\title{
The validation of assays used to measure biomarkers in exhaled breath condensate
}

\section{To the Editors:}

We read with interest the article by Rosias et al. [1] in a recent issue of the European Respiratory Journal. The authors attempted to validate a number of assays using exhaled breath condensate. The values described by Rosias et al. [1] were very similar to those reported by many laboratories, but our interpretation of the data is different. Our experience in validating similar assays in this medium suggests that measuring mediators at these low concentrations is problematic and, as such, we have the following concerns with the article which other researchers need to be aware of.

First, Rosias et al. [1] based coefficient of variation (CV) on a mean of spiked samples, where the spike was far in excess of the measured values. The CVs given were based upon the sample plus spike, rather than the sample alone. In the majority of assays, the working range produces $\mathrm{CVs}$ which are $<15 \%$ [2]. However, in our experience, at low concentrations, the CVs become much higher due to the nonlinearity of the assay [3]. Providing the CVs of spiked samples artificially lowers the $\mathrm{CV}$, and does not give a true representation of the inherent variability of measuring mediators near the lower levels of detection of the assay.

Secondly, the authors stated that "The use of CV is not always the ideal way to express variability: when mean values are low, CV values can be abnormally high" [1]. In our experience, increased CVs at low levels of mediators are a genuine reflection of the increased variability of the assay (signal to noise ratio). At low concentrations, assays often become nonlinear and, since all results are based on interpolation, the CV will, by nature, be inherently high [3]. Figure 1 demonstrates a typical ELISA optical density versus concentration curve for secretory leukoprotease inhibitor plotted using the CVs observed for the assay at varying concentrations of protein. The figure clearly demonstrates that where the assay becomes nonlinear, the CVs increase from $10 \%$ to up to $70 \%$. This truly reflects the variability of the assay in these circumstances.

Thirdly, Rosias et al. [1] suggest that a matrix effect is seen when samples are spiked with $10 \mathrm{pg} \cdot \mathrm{mL}^{-1}$ of mediator, but not at $100 \mathrm{pg} \cdot \mathrm{mL}^{-1}$. This suggests that at low levels of spiking, the components of the sample might interfere with the assay or mediator, which is a recognised phenomenon. However, spiking with $100 \mathrm{pg} \cdot \mathrm{mL}^{-1}$, or even $10 \mathrm{pg} \cdot \mathrm{mL}^{-1}$, is likely to overwhelm and disguise this matrix effect, considering that the actual sample values are between $1-6 \mathrm{pg} \cdot \mathrm{mL}^{-1}$ and so any compounds that interfere with the assay are likely to be low concentrations themselves. If there is a matrix effect at low concentrations, this will have a significant effect on the reliability of the readings produced and should be taken into consideration when using the assay.

Fourthly, the authors speculate that variations in biomarker measurements can be explained by differences in dilution, quality of condensate, different collection techniques, sample processing, storage conditions and analytical techniques. Although all of this may be true, using the low concentrations cited by Rosias et al. [1] we have described wide variability in a single sample, principally due to the variability within the assay [3]. We do not feel that it is prudent to comment on other sources of variability when the variability of the assay itself is so high.

Finally, the authors do not define the lower limit of quantification for any assay. The lower limit of detection defines the difference from zero but is not able to predict where accurate measurements begin. Lower limits of quantification defines the concentration where the assay is linear, and where CVs and spike returns are known and acceptable for the given assay [4].

We feel that the lower limit of quantification is central to the measurement of mediators at these concentrations within this matrix and using current assay methodology suggests that exhaled breathe condensate is not an ideal collection method for protein measurements [3]. Whether the reliability of more

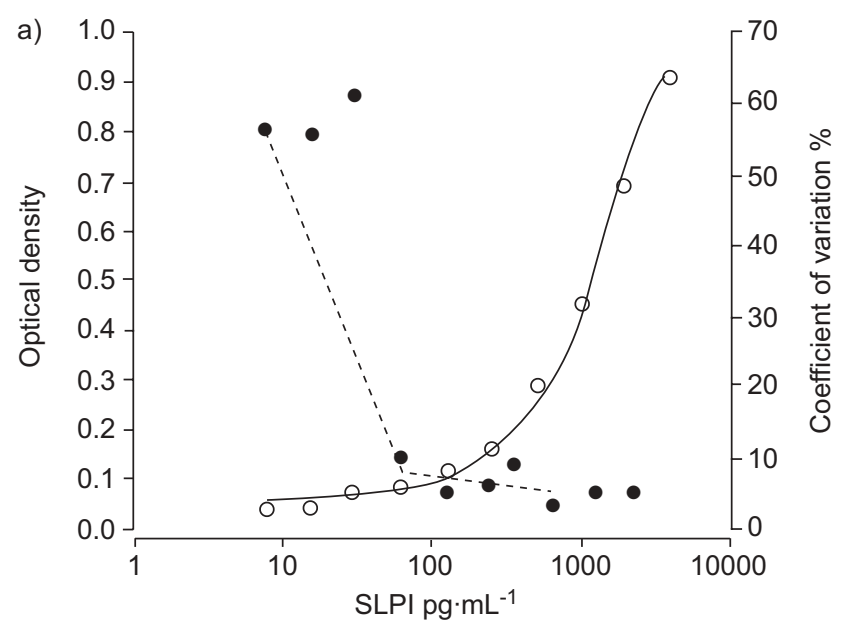

FIGURE 1. A typical ELISA optical density $(O)$ versus concentration curve for secretory leukoprotease inhibitor (SLPI). The typical sigmoid curve is demonstrated $(-$ ) together with the coefficient of variation $(\bullet)$ of sample measurements at different SLPI concentrations. A major increase in the variability of the repeated measures is seen for the plateau of the curve (- - - ). 
sensitive proteomics methodologies overcomes these issues remains to be seen.

\author{
E. Sapey*, D. Bayley, A. Ahmad and R. Stockley \\ *Dept of Medicine, University of Birmingham, , and ${ }^{\#}$ Queen \\ Elizabeth Hospital, Queen Elizabeth Medical Centre, \\ Birmingham, UK.
}

\section{STATEMENT OF INTEREST}

None declared.

\section{REFERENCES}

1 Rosias PP, RobroeksCM, Kester A, et al, Biomarker reproducibility in exhaled breath condensate collected with different condensates. Eur Respir J 2008; 31: 934-942.

2 Stockley RA, Bayley DL. Validation of assays for inflammatory mediators in sputum. Eur Respir J 2000; 15: 778-781.

3 Bayley DL, Abusriwil H, Ahmad A, Stockley RA. Validation of assays for inflammatory mediators in exhaled breathe condensate. Eur Respir J 2008; 31: 943-948.

4 Leung TF, Li CY, Yung E, Liu EK, Lam CW, Wong GW. Clinical and technical factors affecting $\mathrm{pH}$ and other biomarkers in exhaled breathe condensate. Pediatr Pulmonol 2006; 41: 87-94.

DOI: $10.1183 / 09031936.00088608$

\section{From the authors:}

We read with great interest the letter from E. Sapey and coworkers on "The validation of assays used to measure biomarkers in exhaled breath condensate". Indeed, we agree with E. Sapey and co-workers that the variability of current assays can be further improved.

However, in our opinion, this does not imply that "exhaled breathe condensate is not an ideal collection method for protein measurements". One should be aware of the associated ambiguity. There are in fact two relatively separate methods that are involved in the technique of exhaled breath condensate. The first is the method used to collect the condensate of a patient and, subsequently, the method to analyse this condensate.

Hence, we want to highlight that the validation of an assay was not the aim of our study [1]. Moreover, the principal aim was to assess differences between the condensers, including the new glass condenser (i.e. to assess the reproducibility of exhaled breath condensate volume, hydrogen peroxide, 8isoprostane and cytokine measurements using different condensers). Therefore, the coefficients of variation of the cytokines presented in our study [1] do not refer to the intraassay variation, but to the total variability of the cytokines in exhaled breath condensate, which includes intra-assay variation, instrument variability and biological variability in healthy individuals. We hypothesised that exhaled breath condensate collection may be optimised by using a condenser with minimal adhesive properties, and as a result, this may improve the reproducibility of biomarker measurements in exhaled breath condensate. Logically, we had to use an assay to measure hydrogen peroxide, 8-isoprostane and cytokines and we acknowledged the limitations associated with the current assays. However, in order to minimise, or to equally disperse this analytical influence, we used only one type of assay for the measurement of one specific biomarker in exhaled breath condensate that was collected in different ways using different types of condenser. Using this method, we reported significant differences between different methods of condensate collection, in favour of the new glass condenser design [1].

We do not fully agree with E. Sapey and co-workers that the mean of the spiked samples in our study were in excess of measured values. In the case of 8-isoprostane, the mean concentration in exhaled breath condensate for the new condenser was $3.6 \mathrm{pg} \cdot \mathrm{mL}^{-1}$, whereas the spiking concentrations were 3.9 and $7.8 \mathrm{pg} \cdot \mathrm{mL}^{-1}$. For the cytokines, spiking was performed with a concentration of $10 \mathrm{pg} \cdot \mathrm{mL}^{-1}$, whereas the mean values of cytokines ranged $0.7-6.3 \mathrm{pg} \cdot \mathrm{mL}^{-1}$ (new condenser). Therefore, we feel that the intra-assay variation only accounts for a part of the total variability of biomarkers in exhaled breath condensate. The suggestion by E. Sapey and coworkers to define the lower limit of quantification for an assay is an interesting one.

By consequence, and in our opinion, this implicates that the potential or future value of exhaled breath condensate not only depends upon the validity of the analytical technique, but also depends upon the validity of the condensate collection technique, and both of these techniques are prone to improvement.

\section{P.P. Rosias** and E. Dompeling*}

*Dept of Paediatric Pulmonology, University Hospital Maastricht, Care and Public Health Research Institute, Maastricht, and "Dept of Paediatrics, Maasland Hospital, Sittard, Utercht, The Netherlands.

\section{STATEMENT OF INTEREST}

None declared.

\section{REFERENCES}

1 Rosias PP, Robroeks CM, Kester A, et al. Biomarker reproducibility in exhaled breath condensate collected with different condensers. Eur Respir J 2008; 31: 934-942.

DOI: 10.1183/09031936.00101608 